\title{
Singlet Extensions of the MSSM with $\mathbb{Z}_{4}^{R}$ Symmetry
}

\author{
Michael Ratz ${ }^{1}$ and Patrick K. S. Vaudrevange ${ }^{2,3}$ \\ ${ }^{1}$ Physik-Department T30, Technische Universität München, James-Franck-Straße 1, 85748 Garching, Germany \\ ${ }^{2}$ Excellence Cluster Universe, Boltzmannstraße 2, 85748 Garching, Germany \\ ${ }^{3}$ Arnold Sommerfeld Center for Theoretical Physics, Ludwig-Maximilians-Universität München, Theresienstraße 37, \\ 80333 München, Germany
}

Correspondence should be addressed to Patrick K. S. Vaudrevange; patrick.vaudrevange@tum.de

Received 3 March 2015; Accepted 9 June 2015

Academic Editor: Kai Schmidt-Hoberg

Copyright (C) 2015 M. Ratz and P. K. S. Vaudrevange. This is an open access article distributed under the Creative Commons Attribution License, which permits unrestricted use, distribution, and reproduction in any medium, provided the original work is properly cited. The publication of this article was funded by SCOAP ${ }^{3}$.

We discuss singlet extensions of the MSSM with $\mathbb{Z}_{4}^{R}$ symmetry. We show that holomorphic zeros can avoid a potentially large coefficient of the term linear in the singlet. The emerging model has both an effective $\mu$ term and a supersymmetric mass term for the singlet $\mu_{N}$ which are controlled by the gravitino mass. The $\mu$ term turns out to be suppressed against $\mu_{N}$ by about one or two orders of magnitude. We argue that this class of models might provide us with a solution to the little hierarchy problem of the MSSM.

\section{Purpose of This Paper}

The $\mathbb{Z}_{4}^{R}$ symmetry $[1,2]$ provides us with compelling solutions of the $\mu$ and proton decay problems of the minimal supersymmetric extension of the standard model (MSSM). This symmetry appears anomalous, but the anomaly is cancelled by the (discrete) Green-Schwarz (GS) mechanism [3] in such a way that it does not spoil gauge coupling unification (see, e.g., [4] for a discussion). More precisely, if one extends the MSSM by a symmetry (continuous or discrete) that solves the $\mu$ problem and (i) demands anomaly freedom (while allowing GS anomaly cancellation), (ii) demands that the usual Yukawa couplings and the Weinberg operator be allowed, (iii) demands consistency with $S O(10)$ grand unification, and (iv) demands precision gauge coupling unification, then this $\mathbb{Z}_{4}^{R}$ is the unique solution [2] (see also [5] for an alternative proof). By relaxing (iii) to consistency with $S U(5)$, one obtains four additional symmetries [6]. Further, $\mathbb{Z}_{4}^{R}$ can be thought of as a discrete remnant of the Lorentz symmetry of compact extra dimensions; that is, it has a simple geometric interpretation and can arise in explicit string-derived models with the precise MSSM matter content [7]. The charge assignment is very simple: MSSM matter superfields have $\mathbb{Z}_{4}^{R}$ charge 1 while the Higgs superfields have 0 , and the superpotential $\mathscr{W}$ carries $R$ charge 2 .

However, if one attempts to construct singlet extensions of the $\mathbb{Z}_{4}^{R}$ MSSM, one faces the problem that the presence of superpotential coupling of the singlet $N$ to the Higgs bilinear $H_{u} H_{d}$ implies that also a linear term in the singlet is allowed by all symmetries. In more detail, since the Higgs bilinear has $\mathbb{Z}_{4}^{R}$ charge 0 , the singlet $N$ needs to carry charge 2 in order to match the $\mathbb{Z}_{4}^{R}$ charge 2 of the superpotential. Then the desired term $\mathscr{W} \subset N H_{u} H_{d}$ is allowed. However, in this case one might expect to have a problematic, unsuppressed linear term in $N$ in the (effective) superpotential,

$$
\mathscr{W}_{\text {eff }} \supset \Lambda^{2} N
$$

with $\Lambda$ of the order of the fundamental scale. In order to forbid this linear term, one may try to add a new symmetry. It is quite straightforward to see that an ordinary symmetry cannot forbid this linear term and be, at the same time, consistent with criteria (i)-(iv) above: in order to forbid the linear term, the singlet $N$ needs to carry a nontrivial charge under the new symmetry. But, as we want the term $\mathrm{NH}_{u} \mathrm{H}_{d}$, this implies that also $H_{u} H_{d}$ carries a nontrivial charge. 
Consequently, the new symmetry would yield a solution to the $\mu$ problem. However, this is not possible: as stated above, one can prove that (under our assumptions) the unique solution to the $\mu$ problem is $\mathbb{Z}_{4}^{R}$, and this symmetry does not forbid the linear term.

In this paper, we take an alternative route and describe how one can get rid of the linear term (1) by employing holomorphic zeros [8] associated with an additional pseudoanomalous $U(1)$ gauge symmetry.

\section{Forbidding the Linear Term in the $\mathbb{Z}_{4}^{R}(\mathbf{G}) \mathbf{N M S S M}$}

2.1. Setup. Consider a singlet extension of the MSSM with a singlet $N$ and an additional $\mathbb{Z}_{4}^{R} \times U(1)_{\text {anom }}$ symmetry. $U(1)_{\text {anom }}$ is pseudoanomalous $U(1)$ symmetry, whose anomaly is cancelled by the GS mechanism. Such $U(1)$ factors often arise in string compactifications and are accompanied by nontrivial Fayet-Iliopoulos (FI) term [9] $\xi$, which arises at 1-loop [10]. The FI term of the $U(1)_{\text {anom }}$ is assumed to be cancelled by a nontrivial vacuum expectation value (VEV) of a "flavon" $\phi$, which carries negative $U(1)_{\text {anom }}$ charge and $\mathbb{Z}_{4}^{R}$ charge 0 . Without loss of generality, we can normalize $U(1)_{\text {anom }}$ such that $\phi$ has charge -1 and $\xi>0$. (Of course, in true string-derived models the situation is usually more complicated: in approximately 500 out of a total of 11940 MSSM-like models from [11] the FI term can be cancelled with one field only. In all other models, one would have to identify $\phi$ with an appropriate monomial of MSSM singlet fields (see Appendix A for details).) For the sake of definiteness, we assume that

$$
\varepsilon:=\frac{\langle\phi\rangle}{M_{P}} \sim \sin \vartheta_{\text {Cabibbo }} \sim 0.2,
$$

where the Planck scale $M_{P}$ is identified with the "fundamental scale." In this case, $U(1)_{\text {anom }}$ can be used as Froggatt-Nielsen symmetry [12] to explain the flavor structure of quarks and leptons. However, this assumption is not crucial for the subsequent discussion, yet this is what one gets in explicit orbifold compactifications of the heterotic string which exhibit the exact MSSM spectrum at energies below the compactification scale.

Further, also the anomaly of $\mathbb{Z}_{4}^{R}$ is assumed to be cancelled by the GS mechanism with the GS axion being contained in the dilaton or another superfield, which we will denote by $S$. Since the mixed $U(1)_{\text {anom }}-G_{\mathrm{SM}}^{2}$ and $\mathbb{Z}_{4}^{R}-G_{\mathrm{SM}}^{2}$ anomalies are universal, the GS mechanism does not interfere with the beautiful picture of MSSM gauge coupling unification (see, e.g., [4]). The "nonperturbative" term $e^{-b S}$ carries the same $\mathbb{Z}_{4}^{R}$ charge as the superpotential, namely, 2. It might be thought of as some nonperturbative hidden sector (see, e.g., [13]). Further, $e^{-b S}$ will also carry positive $U(1)$ anom charge $s>0$ such that holomorphic zeros get lifted by "nonperturbative" terms. More details on the charge of $e^{-b S}$ can be found in Appendix B (see, e.g., $[6,14])$. In more detail, we demand that

$$
\mathscr{W}_{\text {hid }} \sim M_{P}^{3}\left(\frac{\phi}{M_{P}}\right)^{s} e^{-b S}
$$

TABLE 1: Charge assignment.

\begin{tabular}{lcccc}
\hline & $\phi$ & $H_{u} H_{d}$ & $N$ & $e^{-b S}$ \\
\hline$U(1)_{\text {anom }}$ & -1 & $h>0$ & $n<0$ & $s>0$ \\
$\mathbb{Z}_{4}^{R}$ & 0 & 0 & 2 & 2 \\
\hline
\end{tabular}

be allowed, which is equivalent to the statement that $e^{-b S}$ carries $U(1)_{\text {anom }}$ charge $s>0$. (Note that $s$ may also be fractional even if the charges of all "fundamental" fields are integer, for instance, if one assumes that $\mathscr{W}_{\text {hid }}$ is given by the Affleck-Dine-Seiberg superpotential [15]. Examples for such terms can be found, e.g., in [13].) $\mathscr{W}_{\text {hid }}$ may be thought of as gaugino condensate [16] or some other nonperturbative physics, such as the one discussed in [17], which is involved in spontaneous supersymmetry breaking. We discuss this in more detail in Appendix B. Inserting the $\phi$ VEV we obtain

$$
\mathscr{W}_{\text {hid }} \stackrel{\phi \rightarrow \phi}{\longrightarrow} M_{P}^{2} m_{3 / 2}
$$

in Planck units. (Note that (3) is not the "full" hidden sector superpotential. One must, of course, make sure that $\phi$ does not attain an $F$-term VEV, and one needs to cancel the vacuum energy. A detailed discussion of these issues is, however, beyond the scope of the present paper.) This implies, in particular, that

$$
\left\langle e^{-b S}\right\rangle \sim \frac{m_{3 / 2}}{M_{P}} \varepsilon^{-s} .
$$

That is, $R$ symmetry breaking is controlled by the gravitino mass, as it should be, and due to the presence of $U(1)_{\text {anom }}$ we obtain a Froggatt-Nielsen-like [12] modification of the terms. However, in contrast to the usual Froggatt-Nielsen mechanism, it yields in our setup an enhancement rather than a suppression factor for the lifting of the holomorphic zeros by nonperturbative effects.

2.2. Charges and Allowed Terms in the Superpotential. We summarize the $U(1)_{\text {anom }}$ and $\mathbb{Z}_{4}^{R}$ charges in Table 1 .

Below the $U(1)_{\text {anom }}$ breaking scale set by the $\phi \mathrm{VEV}$, we wish to have a nontrivial $\mu$ term at the nonperturbative level; that is,

$$
\mathscr{W}_{\text {eff }} \supset M_{P} e^{-b s}\left(\frac{\phi}{M_{P}}\right)^{s+h} H_{u} H_{d}
$$

This implies

$$
s+h \geq 0
$$

We will then get effectively

$$
\begin{array}{r}
\mathscr{W}_{\text {eff }} \supset M_{P} e^{-b s}\left(\frac{\langle\phi\rangle}{M_{P}}\right)^{s+h} H_{u} H_{d}=: \mu H_{u} H_{d} \\
\text { with } \mu \sim m_{3 / 2} \varepsilon^{h} .
\end{array}
$$

Next, we wish to couple the singlet $N$ to the Higgs bilinear. We hence demand that

$$
n+h \geq 0
$$


such that

$$
\begin{aligned}
\mathscr{W}_{\text {eff }} & \supset\left(\frac{\langle\phi\rangle}{M_{P}}\right)^{n+h} N H_{u} H_{d} \sim \varepsilon^{n+h} N H_{u} H_{d} \\
= & \lambda N H_{u} H_{d} \quad \text { with } \lambda \sim \varepsilon^{n+h} .
\end{aligned}
$$

Now we wish to forbid the linear term in $N$ at the perturbative level. This can be achieved with holomorphic zeros [8], which amounts in our setup to demanding that

$$
n \stackrel{!}{<} 0 .
$$

This implies, in particular, that the cubic term in $N$ is also forbidden.

Of course, this all works only if we make sure that $\phi$ rather than $N$ cancels the FI term. This might be achieved by postulating that the soft mass squared of $N$ is positive while the one of $\phi$ is negative; that is,

$$
\begin{aligned}
& \widetilde{m}_{\phi}^{2}<0, \\
& \widetilde{m}_{N}^{2}>0 .
\end{aligned}
$$

Full justification of such an assumption would require deriving the setting from some UV complete construction such as a string model. This is, however, beyond the scope of this paper.

We further obtain nonperturbative terms which are linear or quadratic in $N$ if $n+2 s \geq 0$ or $2 n+s \geq 0$, respectively. Altogether we have

$$
\begin{aligned}
& n+h \geq 0 \\
& \Longleftrightarrow \text { coupling } \lambda \text { between } N \text { and } H_{u} H_{d} \text { with } \lambda \sim \varepsilon^{n+h} \text {, } \\
& n<0 \Longleftrightarrow \text { suppress linear term in } N \text {, } \\
& s+h \geq 0 \\
& \Longleftrightarrow \mu \text { term with } \mu \sim M_{P} \varepsilon^{s+h} e^{-b s} \sim \varepsilon^{h} m_{3 / 2}, \\
& \Longleftrightarrow f^{2} N \text { term with } f \sim M_{P} \varepsilon^{(n+2 s) / 2} e^{-b S} \sim \varepsilon^{n / 2} m_{3 / 2}, \\
& \Longleftrightarrow \mu_{N} N^{2} \text { term with } \mu_{N} \sim M_{P} \varepsilon^{2 n+s} e^{-b S} \sim \varepsilon^{2 n} m_{3 / 2}, \\
& \Longleftrightarrow \kappa N^{3} \text { term with } \kappa \sim \varepsilon^{3 n+2 s}\left(e^{-b S}\right)^{2} \sim \varepsilon^{3 n} \frac{m_{3 / 2}^{2}}{M_{P}^{2}},
\end{aligned}
$$

There are many possible values that satisfy all the constraints; for instance, $\{n, h, s\}=\{-1,1,2\}$, which gives us

$$
\begin{aligned}
\lambda & \sim \mathcal{O}(1), \\
\mu & \sim \varepsilon m_{3 / 2}, \\
\mu_{N} & \sim \frac{1}{\varepsilon^{2}} m_{3 / 2}, \\
f & \sim \frac{1}{\sqrt{\varepsilon}} m_{3 / 2} .
\end{aligned}
$$

That is, the (holomorphic) $\mu$ term is roughly two orders of magnitude smaller than $\mu_{N}$, which might be favorable in view of the so-called "little hierarchy problem."

Note also that the effective superpotential

$$
\mathscr{W}_{\text {eff }}=f^{2} N+\mu H_{d} H_{u}+\lambda N H_{d} H_{u}+\mu_{N} N^{2}
$$

admits two solutions to the $F$ - and $D$-term equations, the first one being (recall that $n<0$ )

$$
\begin{aligned}
\langle N\rangle & =-\frac{\mu}{\lambda} \sim-\varepsilon^{|n|} m_{3 / 2}, \\
\left\langle H_{u}\right\rangle & =\left\langle H_{d}\right\rangle=\frac{\sqrt{2 \mu \mu_{N}-\lambda f^{2}}}{\lambda} \sim \varepsilon^{-h / 2} m_{3 / 2} .
\end{aligned}
$$

Here one has electroweak symmetry breaking prior to supersymmetry breaking, and the Higgs VEV may be subject to cancellations since both $\mu \mu_{N}$ and $\lambda f^{2}$ are of the order $\varepsilon^{2 n+h}$, for example, $\varepsilon^{-1}$ in our example. The second solution is

$$
\begin{aligned}
\langle N\rangle & =-\frac{f^{2}}{2 \mu_{N}} \sim-\frac{1}{2} \varepsilon^{|n|} m_{3 / 2}, \\
\left\langle H_{u}\right\rangle & =\left\langle H_{d}\right\rangle=0
\end{aligned}
$$

with unbroken electroweak symmetry for unbroken supersymmetry.

2.3. Discussion. In summary, we find that the $\mathbb{Z}_{4}^{R} \times U(1)_{\text {anom }}$ charge assignment of Table 1 yields an effective superpotential,

$$
\mathscr{W}_{\text {eff }}=f^{2} N+\mu H_{d} H_{u}+\lambda N H_{d} H_{u}+\mu_{N} N^{2},
$$

with all the dimensionful parameters $\mu, \mu_{N}$, and $f$ of the order of the gravitino mass $m_{3 / 2}$. This description is valid below the $U(1)_{\text {anom }}$ breaking scale, which is set by the flavon VEV $\langle\phi\rangle$. In particular, the linear term in the singlet is sufficiently suppressed. In contrast to the original (G)NMSSM [18], here,

(i) there is (essentially) no cubic term in $\mathrm{N}$;

(ii) there is a suppressed linear term in $N$. (Note that, unlike in [18], we cannot shift the singlet in order to eliminate the linear term because the point $N=0$ is special as it denotes the point of unbroken $\mathbb{Z}_{4}^{R}$.) 
The scheme leads to certain predictions and expectations:

(1) Forbidding the linear term by holomorphic zeros implies the absence of a perturbative cubic term in $N$.

(2) Further, we obtain the "little hierarchies" (recall that $n<0$ )

$$
\begin{aligned}
& \mu \sim \varepsilon^{h+2|n|} \mu_{N}, \\
& f \sim \frac{\mu}{\varepsilon^{h+|n| / 2}} .
\end{aligned}
$$

2.4. Further Applications. Clearly, this method of avoiding a linear term in a gauge singlet may find further applications. For instance, in model building one sometimes introduces socalled "driving fields" in order to "explain" a certain structure of flavon VEVs. Here, one may forbid too large tadpole terms in the same way as we have discussed above.

\section{Discussion}

We have discussed how to build singlet extensions of the MSSM with $\mathbb{Z}_{4}^{R}$ symmetry. We have shown that a potentially large linear term in the singlet can be avoided by using holomorphic zeros. The resulting model has a $\mu$ term, a supersymmetric mass of the order of the gravitino mass $m_{3 / 2}$, as well as a coefficient of an effective linear term in the singlet of the order $m_{3 / 2}^{2}$. $\mu$ is expected to be one or two orders of magnitude smaller than $\mu_{N}$. This might be viewed as the first step towards a solution to the little hierarchy problem; that is, explain why the electroweak scale is at least one order of magnitude smaller than the soft supersymmetric terms. Obtaining a complete solution requires the derivation of our setting from a UV complete model, which allows us to compute various terms precisely. This, however, is beyond the scope of this paper.

\section{Appendices}

\section{A. Cancellation of the FI Term}

In this appendix, we discuss how the FI term gets cancelled by a single monomial $\mathscr{M}$. The generalization to the case of several monomials is straightforward. We consider a monomial of chiral superfields $\phi_{i}$, which are assumed to be standard model singlets,

$$
\mathscr{M}=\prod_{i} \phi_{i}^{n_{i}}
$$

with $n_{i} \in \mathbb{N}$. $\mathscr{M}$ is constructed to be gauge invariant with respect to all gauge symmetries except the "anomalous" $U(1)_{\text {anom. In a supersymmetric vacuum one then has }}$

$$
\frac{\left|\left\langle\phi_{i}\right\rangle\right|}{\sqrt{n_{i}}}=v
$$

where $v$ is determined from the requirement that the FI term $\xi>0$ in the $D$-term potential of the anomalous $U(1)_{\text {anom }}$ gets cancelled. That is,

$$
\begin{aligned}
0 & \stackrel{!}{=} D_{\text {anom }}=\xi+\sum_{i} Q_{\text {anom }}^{(i)}\left|\left\langle\phi_{i}\right\rangle\right|^{2} \\
& =\xi+v^{2} \sum_{i} Q_{\text {anom }}^{(i)} n_{i} ;
\end{aligned}
$$

that is,

$$
v=\sqrt{-\frac{\xi}{\sum_{i} Q_{\text {anom }}^{(i)} n_{i}}} .
$$

On the other hand, the "anomalous" charge of the monomial $\mathscr{M}$ is

$$
Q_{\text {anom }}(\mathscr{M})=\sum_{i} Q_{\text {anom }}^{(i)} n_{i}<0
$$

Hence, we obtain

$$
\left|\left\langle\phi_{j}\right\rangle\right|=\sqrt{n_{j}} \sqrt{-\frac{\xi}{Q_{\text {anom }}(\mathscr{M})}} .
$$

That is, if one compares the cases in which (i) the FI term $\xi$ is cancelled by a single field and (ii) the FI term is cancelled by a monomial, there are $\sqrt{n_{j}}$ factors that enhance the flavon VEVs somewhat in case (ii).

\section{B. Nonperturbative Terms in the Superpotential}

In this appendix we discuss how to compute the $U(1)_{\text {anom }}$ charge of the nonperturbative term $e^{-b S}$ in the case that the anomaly of $U(1)_{\text {anom }}$ is cancelled via the universal GreenSchwarz mechanism. We follow the notation of Appendix A.2 in [6].

The Kähler potential of the dilaton $S$ reads

$$
K\left(S, S^{\dagger}, V\right)=-\ln \left(S+S^{\dagger}-\delta_{\mathrm{GS}} V\right)
$$

Then, under $U(1)_{\text {anom }}$ gauge transformations with gauge parameter $\Lambda(x)$, the $U(1)_{\text {anom }}$ vector field $V$ and the dilaton $S$ shift according to

$$
\begin{aligned}
V & \mapsto V+\frac{i}{2}\left(\Lambda(x)-\Lambda(x)^{\dagger}\right), \\
S & \mapsto S+\frac{i}{2} \delta_{\mathrm{GS}} \Lambda(x),
\end{aligned}
$$

such that $K\left(S, S^{\dagger}, V\right)$ is invariant. Furthermore, in order to cancel the cubic anomaly $A_{U(1) \text { anom }}^{3}$, the constant $\delta_{\mathrm{GS}}$ has to satisfy

$$
\delta_{\mathrm{GS}}=\frac{1}{2 \pi^{2}} A_{U(1)_{\mathrm{anom}}^{3}}=\frac{1}{6 \pi^{2}} \operatorname{tr} Q_{\mathrm{anom}}^{3},
$$


where the trace sums over the $U(1)_{\text {anom }}$ charges of all matter superfields. Consequently, one can define a charge $s$ for the nonperturbative term,

$$
e^{-b S} \mapsto e^{-i s \Lambda(x)} e^{-b S}
$$

with $b>0$ and the charge $s$ is given by

$$
s=Q_{\text {anom }}\left(e^{-b S}\right)=\frac{b}{2} \delta_{\mathrm{GS}}=\frac{b}{12 \pi^{2}} \operatorname{tr} Q_{\mathrm{anom}}^{3} .
$$

Depending on $\operatorname{tr} Q_{\text {anom }}^{3}$ the charge $s$ of $e^{-b S}$ can be positive or negative. On the other hand, in certain string-derived models, in which the Green-Schwarz mechanism is universal, one has the relation

$$
\operatorname{tr} Q_{\text {anom }}^{3}=\frac{1}{8} \operatorname{tr} Q_{\text {anom }}
$$

using the fact that the generator of $U(1)_{\text {anom }}$ is normalized to $1 / 2$. Then one obtains

$$
s=\frac{b}{96 \pi^{2}} \operatorname{tr} Q_{\mathrm{anom}}
$$

We have chosen $U(1)_{\text {anom }}$ such that the FI term $\xi$ is positive; that is,

$$
\xi=\frac{g}{192 \pi^{2}} \operatorname{tr} Q_{\text {anom }}>0
$$

see Appendix A. Consequently, the $U(1)_{\text {anom }}$ charge of the nonperturbative term $e^{-b S}$ is positive as well; that is,

$$
s=\frac{2 b}{g} \xi>0 .
$$

For instance, in the case of a condensing $S U\left(N_{c}\right)$ group with $N_{f}<N_{c}$ fundamental and antifundamental "matter" fields, $Q$ and $\widetilde{Q}$, one has (see, e.g., [13, Equation (2.7)] of the published version)

$$
\begin{aligned}
\mathscr{W}_{\text {hid }} \supset\left(N_{c}-N_{f}\right) \frac{\Lambda^{\left(3 N c-N_{f}\right) /\left(N_{c}-N_{f}\right)}}{(\operatorname{det} M)^{1 /\left(N_{c}-N_{f}\right)}} \\
+\left(\frac{\phi}{M_{P}}\right)^{q+\widetilde{q}} m_{i}^{\bar{\jmath}} M_{\bar{\jmath}}^{i}
\end{aligned}
$$

where $\Lambda$ denotes the renormalization group invariant scale and carries charge $Q_{\text {anom }}(\Lambda)=N_{f}(q+\widetilde{q}) /\left(3 N_{c}-N_{f}\right) \cdot q$ and $\widetilde{q}$ are the "anomalous" charges of $Q$ and $\widetilde{Q}$, respectively. Inserting the VEV of the mesons $M_{\bar{j}}^{i}=Q^{i} \widetilde{Q}_{\bar{j}}$ (see [13, Equation (2.13)]), one obtains a term of the form (3).

\section{Conflict of Interests}

The authors declare that there is no conflict of interests regarding the publication of this paper.

\section{Acknowledgments}

The authors would like to thank Mu-Chun Chen and Graham Ross for useful discussions. Michael Ratz would like to thank the UC Irvine, where part of this work was done, for hospitality. This work was partially supported by the DFG cluster of excellence "Origin and Structure of the Universe" (http://www.universe-cluster.de) by Deutsche Forschungsgemeinschaft (DFG). The authors would like to thank the Aspen Center for Physics for hospitality and support. This research was done in the context of the ERC Advanced Grant Project "FLAVOUR" (267104).

\section{References}

[1] K. S. Babu, I. Gogoladze, and K. Wang, "Natural R-parity, $\mu$ term, and fermion mass hierarchy from discrete gauge symmetries," Nuclear Physics B, vol. 660, no. 1-2, pp. 322-342, 2003.

[2] H. M. Lee, S. Raby, M. Ratz et al., "A unique $\mathbb{Z}_{4}^{R}$ symmetry for the MSSM," Physics Letters B, vol. 694, no. 4-5, pp. 491-495, 2011.

[3] M. B. Green and J. H. Schwarz, "Anomaly cancellations in supersymmetric $D=10$ gauge theory and superstring theory," Physics Letters B, vol. 149, no. 1-3, pp. 117-122, 1984.

[4] M.-C. Chen, M. Fallbacher, and M. Ratz, "Supersymmetric unification and $R$ symmetries," Modern Physics Letters A, vol. 27, no. 40, Article ID 1230044, 15 pages, 2012.

[5] M.-C. Chen, M. Ratz, and V. Takhistov, " $R$ parity violation from discrete $R$ symmetries," Nuclear Physics B, vol. 891, pp. 322-345, 2015.

[6] H. M. Lee, S. Raby, M. Ratz et al., "Discrete $R$ symmetries for the MSSM and its singlet extensions," Nuclear Physics B, vol. 850, no. 1, pp. 1-30, 2011.

[7] R. Kappl, B. Petersen, S. Raby, M. Ratz, R. Schieren, and P. K. Vaudrevange, "String-derived MSSM vacua with residual $R$ symmetries," Nuclear Physics B, vol. 847, no. 2, pp. 325-349, 2011.

[8] M. Leurer, Y. Nir, and N. Seiberg, "Mass matrix models," Nuclear Physics B, vol. 398, no. 2, pp. 319-342, 1993.

[9] P. Fayet and J. Iliopoulos, "Spontaneously broken supergauge symmetries and goldstone spinors," Physics Letters B, vol. 51, no. 5, pp. 461-464, 1974.

[10] W. Fischler, H. P. Nilles, J. Polchinski, S. Raby, and L. Susskind, "Vanishing renormalization of the $D$ term in supersymmetric U(1) theories," Physical Review Letters, vol. 47, article 757, 1981.

[11] H. P. Nilles and P. K. S. Vaudrevange, "Geography of fields in extra dimensions: string theory lessons for particle physics," in Perspectives on String Phenomenology, vol. 22 of Advanced Series on Directions in High Energy Physics, World Scientific, 2015.

[12] C. D. Froggatt and H. B. Nielsen, "Hierarchy of quark masses, cabibbo angles and CP violation," Nuclear Physics B, vol. 147, no. 3-4, pp. 277-298, 1979.

[13] P. Binetruy and E. Dudas, "Gaugino condensation and the anomalous $U(1)$," Physics Letters B, vol. 389, no. 3, pp. 503-509, 1996.

[14] N. Arkani-Hamed, M. Dine, and S. P. Martin, "Dynamical supersymmetry breaking in models with a Green-Schwarz mechanism," Physics Letters B, vol. 431, no. 3-4, pp. 329-338, 1998.

[15] I. Affleck, M. Dine, and N. Seiberg, "Dynamical supersymmetry breaking in supersymmetric QCD," Nuclear Physics, Section B, vol. 241, no. 2, pp. 493-534, 1984. 
[16] H. P. Nilles, "Dynamically broken supergravity and the hierarchy problem," Physics Letters B, vol. 115, no. 3, pp. 193-196, 1982.

[17] K. Intriligator, N. Seiberg, and D. Shih, "Dynamical SUSY breaking in meta-stable vacua," Journal of High Energy Physics, vol. 2006, article 21, 2006.

[18] M. Drees, "Supersymmetric models with extended Higgs sector," International Journal of Modern Physics A, vol. 4, no. 14, pp. 3635-3651, 1989. 

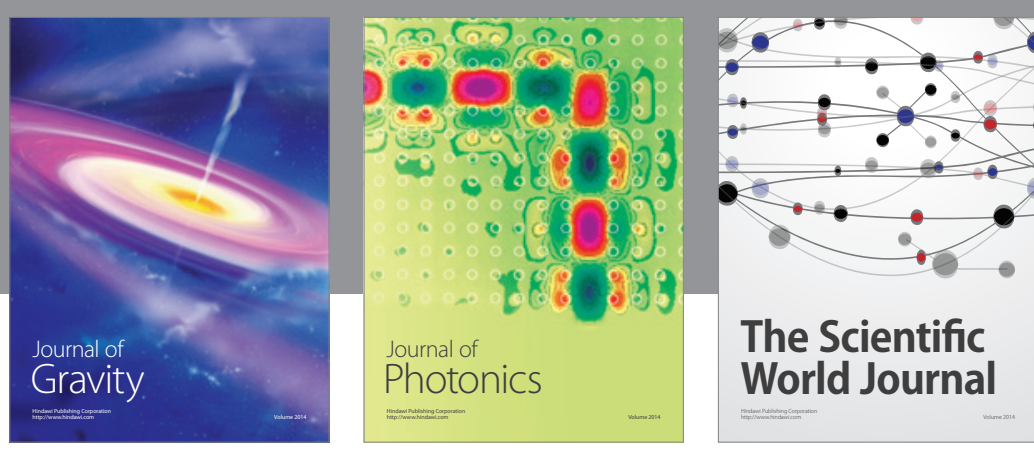

The Scientific World Journal
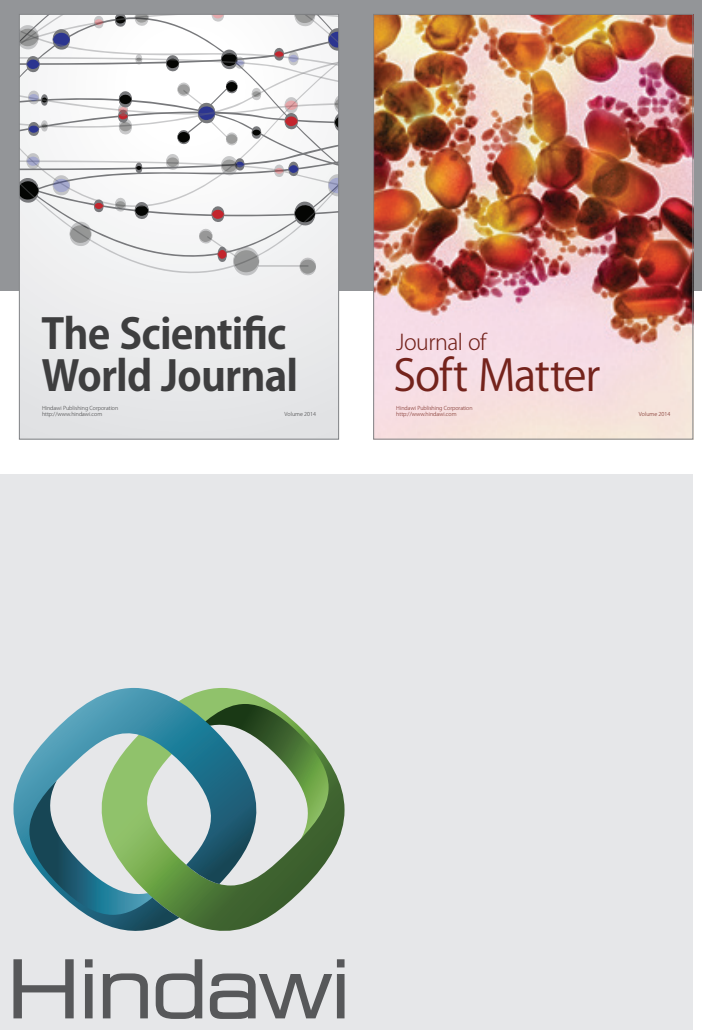

Submit your manuscripts at

http://www.hindawi.com

nternational Journal of

Statistical Mechanics
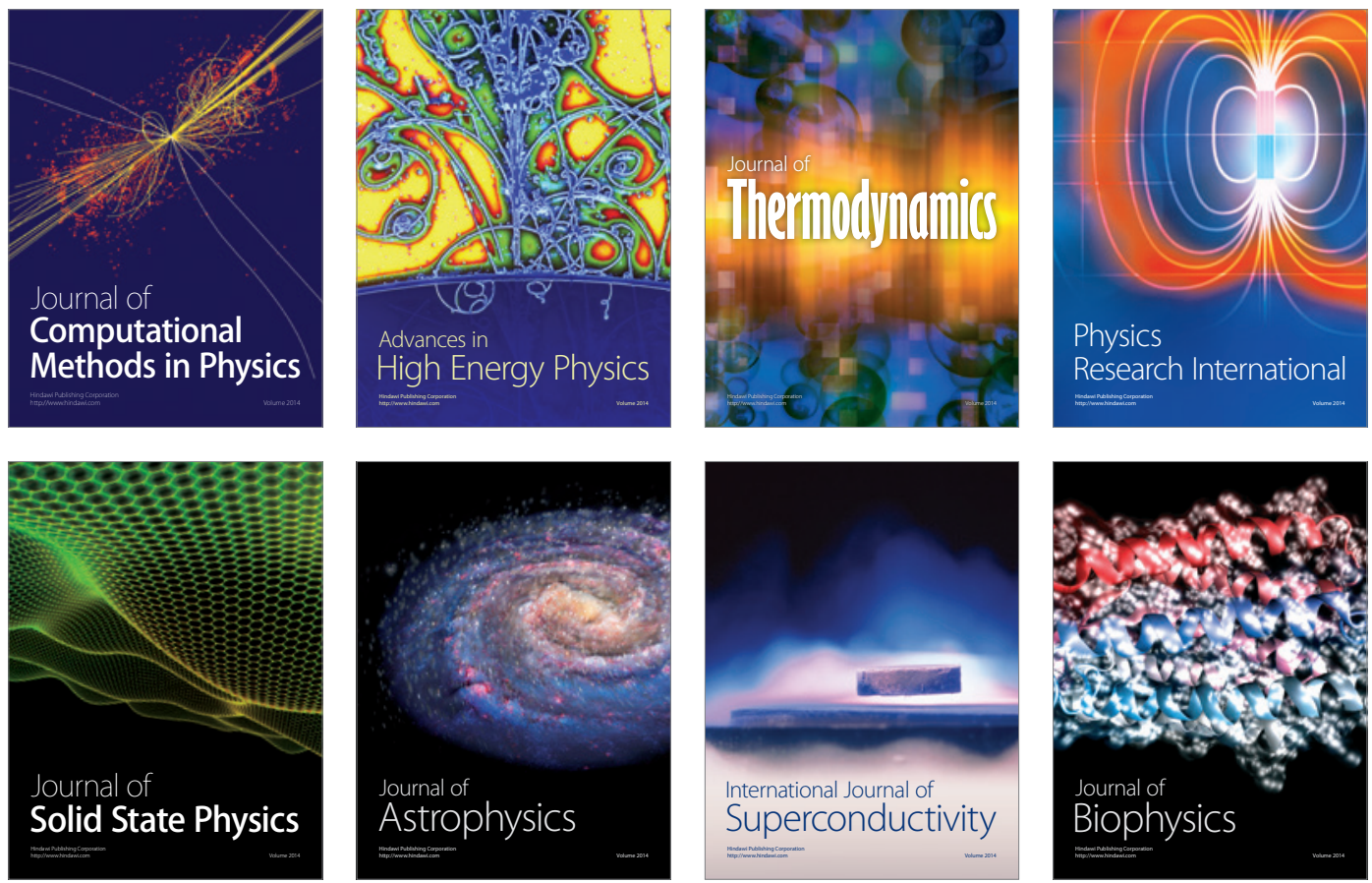
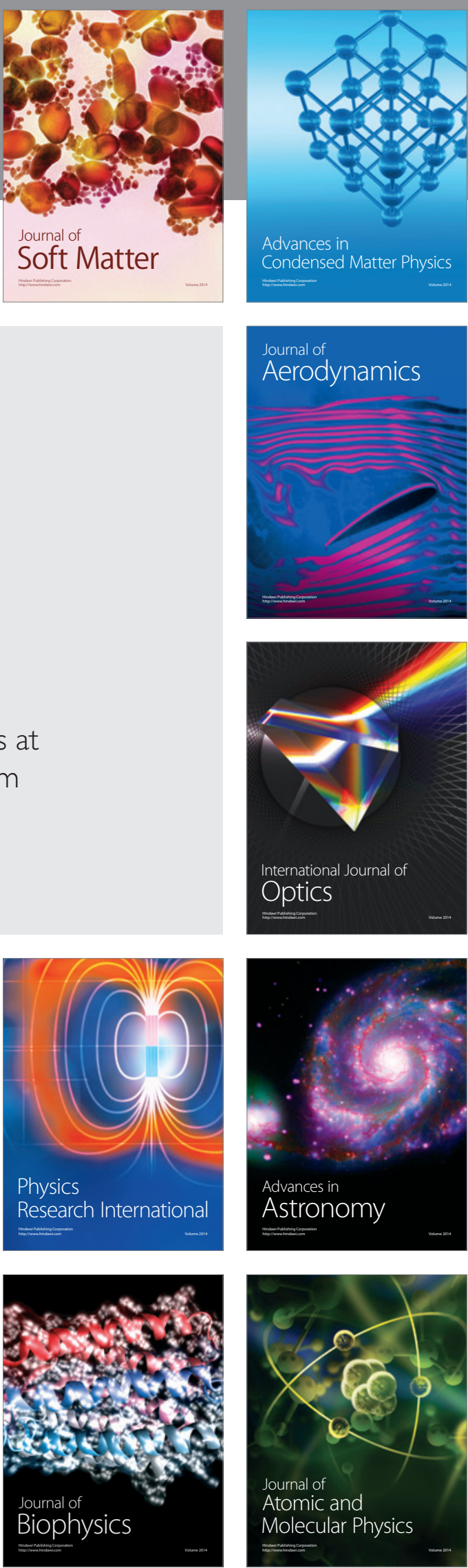